\title{
THE ANALYTIC HIERARCHY PROCESS (AHP) AND THE USER NEED ELICITATION IN THE HEALTH TECHNOLOGY ASSESSMENT (HTA)
}

\author{
Leandro Pecchia* \\ Electrical Systems and Optics Research Division, \\ Faculty of Engineering, \\ University of Nottingham, \\ Nottingham, UK \\ Email: leandro.pecchia@nottingham.ac.uk, \\ Stephen Morgan \\ Electrical Systems and Optics Research Division, \\ Faculty of Engineering, \\ University of Nottingham, \\ Nottingham, UK \\ Email: steve.morgan@nottingham.ac.uk
}

\begin{abstract}
The use of scientific quantitative methods to support decision making is becoming necessary in healthcare systems, especially for Health Technology Assessment (HTA), which is a particularly complex decision making processes. This paper, after introducing three case studies in which the AHP was used to elicit user needs, generalize the method employed, provides recommendations and discusses critical methodological aspects, which the authors consider crucial when applying AHP to user need elicitation studies in HTA. The users of medical devices can include patients, healthcare professionals (with very different specializations), hospital managers, politician and general public. The AHP proved to be an effective method to elicit user needs, especially if some adjustments to the hierarchy design and to the questionnaires layout are followed.
\end{abstract}

Keywords: AHP, HTA, user need elicitation

\section{Introduction}

The use of scientific quantitative methods to support decision-making is becoming necessary in healthcare systems. Healthcare personnel (HCP) are committed to follow the best available evidence in terms of the hierarchy of evidence, following the recommendations of Evidence Based Medicine (EBM), which relies on well-designed trials (Bracale, Rovani et al. 2012), meta-analyses (Bracale, Rovani et al. 2011) or network meta-analyses (Bracale, Rovani et al. 2012), leaving few space for qualitative knowledge and clinicians experience. Nonetheless, despite the hierarchy of evidence, the complexities of medical decision-making requires additional information, regardless of whether it is quantitative or qualitative (Leys 2003). In this scenario, qualitative knowledge is paramount important and have the potentiality to rise the pyramid of evidence if supported by well-structured scientific methods.

The Health Technology Assessment (HTA) is a particularly complex decision making processes, and one that would benefit greatly from the adoption of scientific methods to allow the consideration, as

\footnotetext{
* Corresponding author
} 
part of a coherent framework, a range of different factors, including intangible ones such as user requirements. The AHP has been successfully applied throughout the HTA process, performing especially well for decision-making regarding new technologies as well as in the early stages of medical devices development. In fact, standard methods of HTA do not allow prioritization of user needs or preferences, failing to measure the matching of healthcare technologies and real user needs, failing therefore to inform the manufacturer, which have not sufficient quantitative knowledge regarding requirements that would allow the development of more effective technologies (Pecchia, Bath et al. 2011).

Several studies have already assessed the effectiveness of AHP as a method for medical and healthcare decision-making (Hummel, van Rossum et al. 2000; Liberatore and Nydick 2008). A number of articles have highlighted the advantages in supporting hospital purchases with AHP, which is valuable for multidimensional and multifactor decisions (Sloane, Liberatore et al. 2003), and is easy to use (Chatburn and Primiano 2001) and time-saving (Turri 1988). Other studies proved that AHP is particularly effective in investigating patients' preferences in selecting the most appropriate medical treatment (Dolan 2010) or to integrate patients' view in HTA (Danner, Hummel et al. 2011). Fewer studies focused user need elicitation to drive the design of new medical devices (Craven, Pecchia et al. 2013; Pecchia, Martin et al. 2013; Pecchia, Martin et al. 2013) and healthcare services (Pecchia, Bath et al. 2011; Pecchia and Craven 2013).

This paper introduces three case studies in which the AHP was used to elicit user needs involving different users as medical doctors (Pecchia, Martin et al. 2013), patients (Pecchia, Martin et al. 2013) or healthy patients (Pecchia, Bath et al. 2011), generalizing the method employed to elicit users' need using AHP. Finally, some heuristic recommendations are given and critical methodological aspects, which the authors consider crucial when applying AHP to user needs elicitations, especially when users are not skilled in the use of complex mathematical methods, are discussed.

\section{Case studies}

This section describes three case studies that will be used to discuss the methodological issues and AHP criticism when responders (the users) are not experienced in the use of AHP. After introducing the object of the three studies and the its research questions, the main categories of the hierarchies developed in the three case studies and relevant information of the responders involved in each study are presented in this section. Further details regarding the three studies mentioned can be easily found in the references, because all the papers relative to these case studies are freely available via internet.

\subsection{AHP to elicit user needs: a case study on a Computed Tomography (CT) (Pecchia, Martin et al. 2013)}

The multi-slice CT scanner refers to a special CT system equipped with a multiple-row detector array to collect simultaneously data at different slice locations. The multi-slice CT scanner has the capability of rapidly scanning a large longitudinal volume with high resolution. There are two modes for a CT scan: step-and-shoot CT or helical (or spiral) CT [46]. In recent years, developments in CT technology have provided increasing temporal and better spatial resolution. Scan times are much shorter and slice thickness much thinner with increasing rotation speed and increasing number of active detector-rows, from 4 and 16 detector rows to 64-detector CT scanners [47]. The different features of this device may significantly affect its costs. For instance, to equip this device with a system for continuous patient monitoring during the examination may be expensive. In addition, the technical performance of the device may strongly vary, affecting the final cost. It is therefore of paramount importance to elicit user needs before the manufacturer develop a new model, especially considering that, given the economical constrains, users are not willing to spend more to have features or performance not strictly required. 
In this case study, users involved were five clinicians (age 54 \pm 5 years, $40 \%$ males), each with more than 20 years of experience, employed in the same medium-sized public hospital but with different clinical specialization and working in wards with different criticisms: radiology unit, emergency unit, minimally invasive ear surgery unit, neurology unit performing both elective and emergency neurosurgery. All the five had experience of different clinical environments, but each was asked to answer in relation to the unit in which they were working at the time of the study. The surgeon from the ear surgery unit was mainly responsible for child ear cochlear implants, which is an elective surgery. Two surgeons answered from the neurology units: one was in charge of emergency neurological surgeries and the other of the elective neurologic surgeries. The hierarchy developed the study is reported in Figure 1. The preliminary results of this study were presented and discussed to the ISAHP2011 (Pecchia, Bath et al. 2011) and the full study is described in a paper (Pecchia, Martin et al. 2013) on an open access journal1.

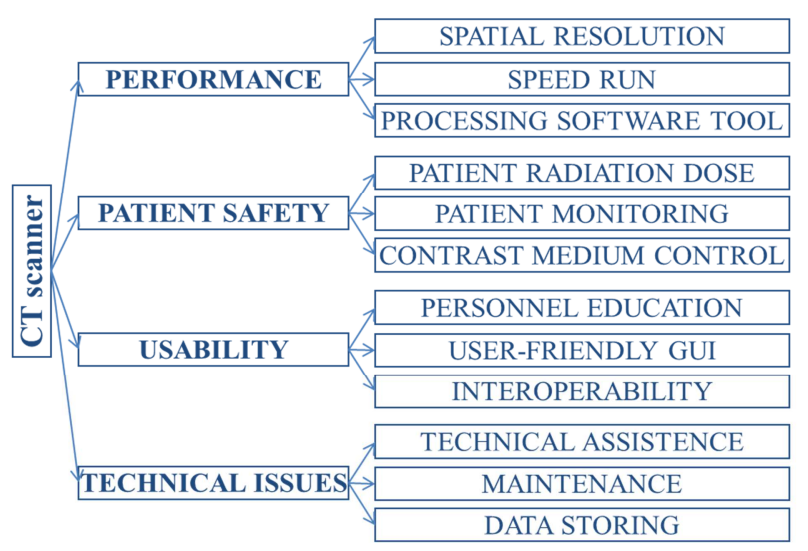

Figure 1. The first two layers of the hierarchy of user needs regarding the user of a CT scanner.

2.2 AHP to elicit user needs: a case study on a device for auto-injection of epinephrine (Pecchia, Martin et al. 2013)

Anaphylaxis is a life threatening allergic reaction, which affects the respiratory and/or cardiovascular systems (Muraro, Roberts et al. 2007). A key component in the treatment of anaphylaxis relies on the patient providing routine self-care and management to prevent this occurring (Choo and Sheikh 2007). Whilst anaphylaxis may be triggered by exposure to latex rubber, insect venom and medication, the most common cause is exposure to foods including peanuts, nuts, fish, milk and eggs (Ewan 1998). The incidence of anaphylaxis has risen dramatically in recent years, as reflected by a sevenfold increase in anaphylaxis-related UK hospital admission between 1990/1 and 2003/4 (Gupta, Sheikh et al. 2007). The treatment of anaphylaxis is a prompt intramuscular injection of epinephrine, typically administered by the patients themselves using Epinephrine Auto-Injectors (EAIs). It is therefore not surprising that prescriptions of EAIs have risen, with 10,700 prescriptions being issued in England in 2001, rising to 21,100 in 2005 (Sheikh, Hippisley-Cox et al. 2008). Patients considered at risk of anaphylaxis are prescribed at least one EAI, which in accordance with self-care best practice for this condition, is to be carried by the patient at all times so that the device is readily available for rapid self-treatment when necessary (Baral and Hourihane 2005). It is widely accepted that not having

\footnotetext{
${ }^{1}$ http://www.biomedcentral.com/1472-6947/13/2
} 
an EAI available at the scene of a severe anaphylaxis event puts the patient at significant risk of a fatal outcome (Pumphrey 2008).

Although EAIs have been designed to be used as self-treatment devices by patients there is evidence to suggest that patients often do not engage in appropriate self-care practices such as the carriage and use of the device when necessary (Cummings, Knibb et al. 2010). A study of fatal anaphylactic reactions revealed that only $10 \%$ of individuals actually had epinephrine to hand when it was required (Bock, Munoz-Furlong et al. 2001), and even when the device is to hand, it is often not used (Simons 2004).

Despite the serious consequences of not having such a device to hand, there is a lack of research that considers the experiences and attitudes of patients and the strategies they use in the delivery of care for this condition. More specifically, to the best of our knowledge, little research has been carried out to specifically explore, from the adult patients' perspective, what patient motivations are for carriage or non-carriage of EAIs and/or their deployment/non-deployment at appropriate times (Money, Barnett et al. 2012).

The study investigated which factors are more relevant in driving he patients in not to carry the EAI with them everyday. More than 30 different user needs were identified in literature and in previous study in which user preferences were elicited using qualitative research methods. These factors were organized in 3 categories and 11 subcategories giving the hierarchy whose first two levels are represented in Fig2.

This study is still running and final responders have not been interviewed yet. This is because during its piloting, which involved 5 patients with different ages, backgrounds and pathology severity, the investigators realized that the heterogeneity of the patients involved was to huge and the study was readdressed to adolescent patients that resulted to have the bigger problems in terms of protocol adherences. This required a different ethical approval.

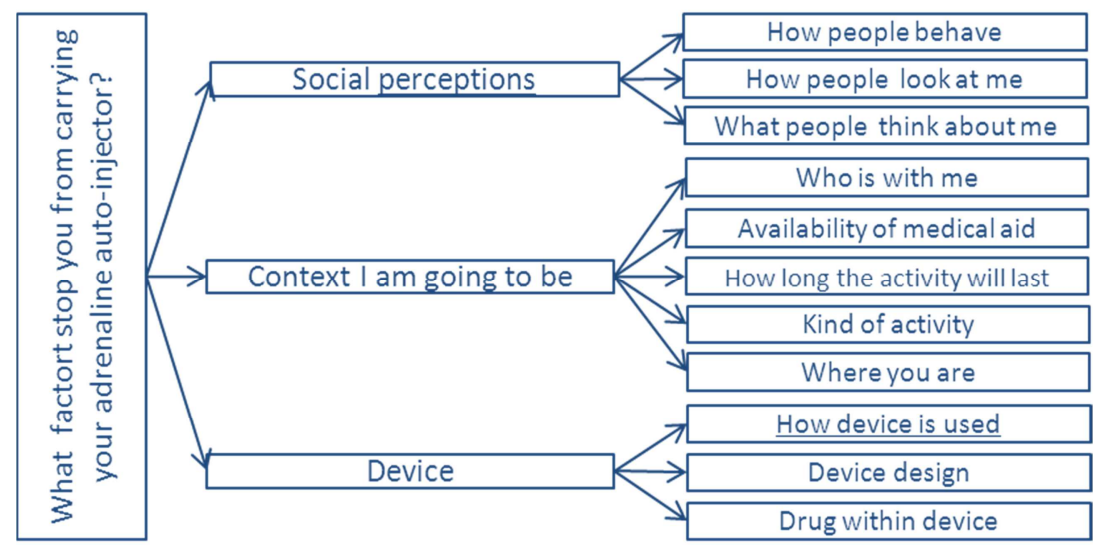

Figure 2. The first two layers of the hierarchy of user needs that refrain patients to carry the EAIs

\subsection{AHP to elicit user needs: a case study on elderly wellbeing (Pecchia, Bath et al. 2011)}

The definition of well-being is complex and well-being may be affected by a wide variety of factors. Among older people, well-being is even more complex, because it may vary depending on different individuals' backgrounds and experiences. Nonetheless, it is important to understand what the concept of well-being means to older people and which factors affect well-being, because of the growing importance of cost-utility studies in medicine and health services research. Such studies aim to measure the quality of life in participants before and after a medical/surgical intervention. However, the scales used to measure quality of life are based on expert opinion, and could be improved by being more focused on what the concept of well-being means to older people themselves. 


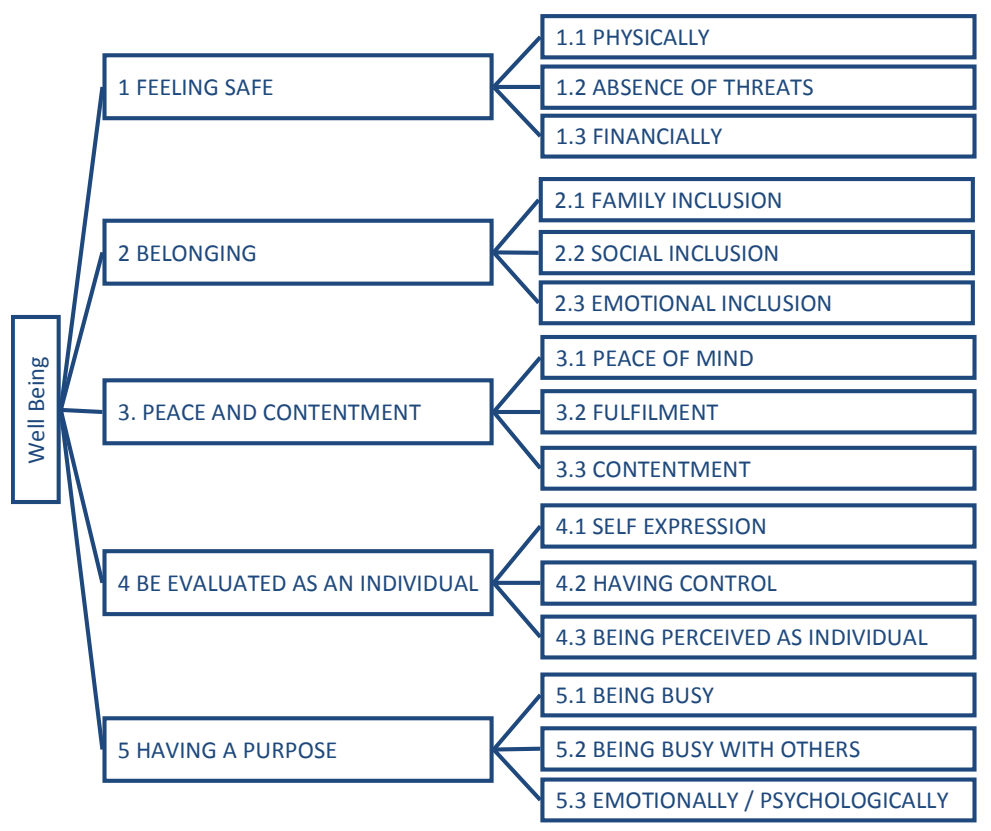

Figure 3. The first two layers of the hierarchy of needs that affect the wellbeing in the elderly In this study, the factors affecting wellbeing in later life have been prioritized. Basing on scientific literature, we defined a hierarchy of 45 factors, organized into 15 sub-categories, which were grouped into 5 main categories. The first two levels of the hierarchy are shown in Figure 3. The questionnaire was submitted to 23 people over 65 years old who participated in a focus group on well-being. The results of this study were presented and discussed to the IASHP2012 in Sorrento and the paper is available online ${ }^{2}$.

\section{Method}

In the case studies introduced above, the AHP method was applied following the 5 steps as shown in Figure 4.

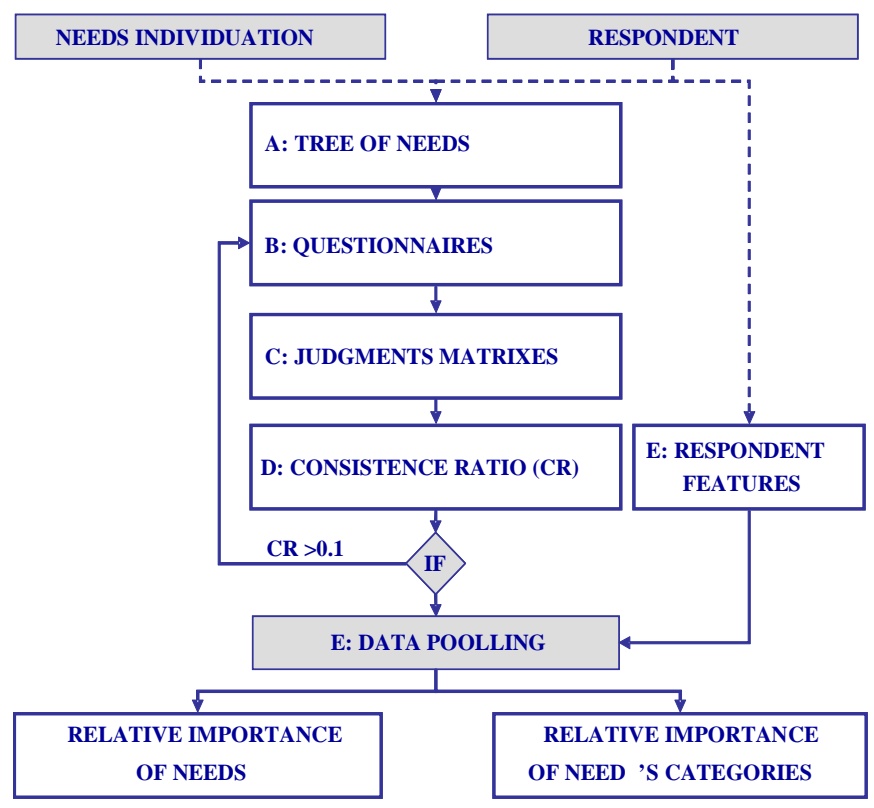

Figure 4: AHP method for user need elicitation.

\footnotetext{
${ }^{2}$ http://204.202.238.22/isahp2011/dati/pdf/126 0121 Pecchia.pdf
} 


\subsection{Needs identification}

Those needs that the healthcare technology aimed to satisfy were identified. This involved from 2 to 4 domain experts and device potential users in 1 or 2 focus groups. The needs were identified using scientific literature and via focus groups.

\subsection{Tree of needs}

A tree of needs with nodes (categories of sub-categories) and leaves (needs) was developed. This involved 2 experts (a domain expert and an expert user of the AHP). This tree was discussed with 1-2 domain experts not involved in its development. The piloting of the developed three with a limited number of users (2-4 users, 1 domain expert, 1 expert in AHP use) took about 3 days.

\subsection{Questionnaires}

Questionnaires were developed to measure needs priority. Some specific considerations due to the low experiences of responders with the AHP methods leaded the design of the questionnaires, as detailed in the next section. The development and the piloting of the questionnaires with a limited number of users (2-4 users, 1 domain expert, 1 expert in AHP use) took about 3 days.

\subsection{Judgments and consistency estimation}

This was performed according to the Saaty theory (Saaty 1988). Since in a user need elicitation study difference in opinions are as relevant as the final priorities, no method of the substitution of inconsistent values were adopted. This because these methods act as a low pass smoothing filters, reducing differences. The inconsistencies were discussed with responders and reduced asking them to re-answer to the same questionnaire. Today here is not evidence on how to choose the minimum number of responders. It is recommended to pay particular attention to those questionnaires sections where the responders are more exposed to lose their attention.

\subsection{Data pooling}

The goal of an elicitation study is to explore difference among different groups of users. For this reason, the final results of the elicitation is not only the relative importance of each need (priority), but the priority of each need in different responders groups. For this reason, global and local priorities were computed applying the geometric mean to the judgment matrices in different groups of responders and not among all of them. Differences were than presented and discussed with responders. In some cases, it was useful to compute the priorities given by each responder and then to compute the arithmetic mean on these value (eigenvectors' components) and not among matrices. This was particularly useful to show standard deviation among priority in different groups, which are masked by geometric mean applied to the judgment matrices.

\section{Methodological considerations for AHP in user need elicitation in healthcare}

To elicit the user needs in healthcare is a particular delicate task, given the heterogeneity of the users, which goes from the patients to the HCP, to personnel with technical backgrounds. Moreover, the patients are often emotionally involved in the elicitation process. In the majority of the case, these users have not familiarity with mathematical methods for decision-making. This is one of the reasons that leaded the AHP to perform very well in user need elicitation. In the same time, some methodological arrangements are needed, in consideration of the responders' peculiar background and role. The following are empirical observation that leaded the AHP studies in consideration of the peculiarity of user need elicitation in healthcare. However, the experience of the authors is that adopting the following arrangements the AHP application was easier and met less problems. 
Regarding the development of the tree of needs, it was important to develop, as much as possible, a symmetric hierarchy: the same number of elements in each node; the same deepness of the hierarchy in each branch of the tree. This allowed then the calculation of global priorities of all the needs. This is not a straightforward process when the hierarchy is not symmetric, and this is because needs falling in a more crowdie node, result relatively less important than those falling in less populated ones. Moreover, it is recommended the use of a limited number of elements per node. Three was particularly effective also to reduce, or just to discuss, inconsistencies.

Regarding the questionnaires, it is necessary to be clear with the questions posed at each one of the survey sections, which correspond to a different tree node. It is important to repeat and adapt the question at each new section of the survey changing it according to the specific category. Considering that in each language there is a default direction for reading and writing (i.e. this paper was written from left to right and from up to down), responders could be biased in judging the first needs under comparisons more relevant than the last ones and the one on the left more important than the one on the right. State clearly that the order of the questions is not meant to be related to the importance of the needs. However, try to put the same number of times each need on the left and on the right of the survey. We found it useful to put comparisons in chain (i.e. AvsB, BvsC, CvsA), more than compare each need with the remaining ones (i.e. AvsB, AvsC, BvsC). This helped also inexperienced responders to be more consistent. Additionally, the order of the questionnaire section went from the lower part of the hierarchy to the upper, asking to prioritize final needs falling in the lower subcategories going up to pairwise comparisons of main need categories. This allows the responder to know exactly what it is in each category.

Moreover, ask one question (Figure 5) asking simultaneously which need is more important and how much, resulted easier and faster than posing two questions.

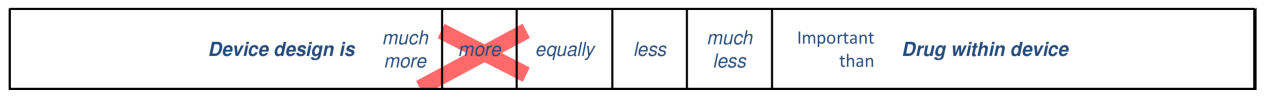

Figure 5. Questionnaire layout.

In the same Figure, it is possible to observe that the judgments are positioned in such an order that the responder is asked to put an $\mathrm{X}$ closer to the need that he consider more important, and as closer as they consider relevant the need under comparison. This gave the same graphical impact of the visual analog scales widely used in medicine to measure patients' symptoms as fatigue or pain (Figure 6). This graphical approach recall dichotomist choices, where the responders are asked to select one of the two options by marking it.

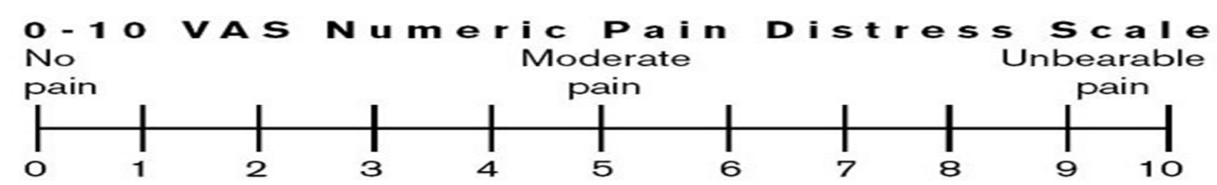

Figure 6. A typical layout of a visual analog scale.

To compare few needs in each section of the questionnaire (three is our preferred number) there is not really the need to use all the 9 steps of the Saaty natural scale. According to our experience, few responders really used all the 9 judgments and 3-5 steps are often sufficient to many responders to prioritize up to 4 elements. Finally, we found paramount important to report under each section of the survey the arm of the hierarchy where the father node of the needs under comparison have been allocated (Figure 7). 


\begin{tabular}{|c|c|c|c|c|c|c|c|}
\hline Expiry date is & $\begin{array}{l}\text { much } \\
\text { more }\end{array}$ & more & equally & less & $\begin{array}{l}\text { much } \\
\text { less }\end{array}$ & $\begin{array}{r}\text { Important } \\
\text { than }\end{array}$ & $\begin{array}{l}\text { Appearance of the liquid } \\
\text { (cloudy/clear) }\end{array}$ \\
\hline $\begin{array}{r}\text { Appearance of the liquid } \\
(\text { cloudy/clear }) \text { is }\end{array}$ & $\begin{array}{l}\text { much } \\
\text { more }\end{array}$ & more & equally & less & $\begin{array}{l}\text { much } \\
\text { less }\end{array}$ & $\begin{array}{r}\text { Important } \\
\text { than }\end{array}$ & How the devices has been stored \\
\hline How the devices has been stored is & $\begin{array}{l}\text { much } \\
\text { more }\end{array}$ & more & equally & less & $\begin{array}{l}\text { much } \\
\text { less }\end{array}$ & $\begin{array}{r}\text { Important } \\
\text { than }\end{array}$ & Expiry date \\
\hline
\end{tabular}

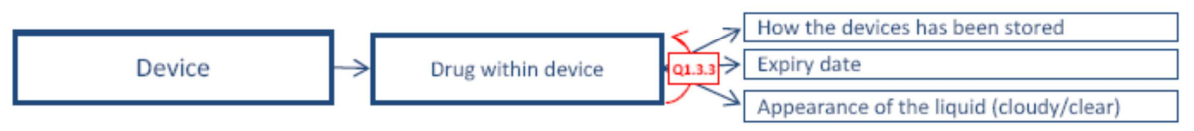

Figure 7. Layout of a section of the questionnaire and representation of the relative arm of the hierarchy, where the node under investigation was located

Regarding priority calculation, according to our experience, the distributed normalization mode (where the sum of the relative priorities is one) is more suitable for user need elicitation. This is mainly because changing the users (medical doctors with different specializations or patients with different age) the priorities of the needs could be very different, so the idea of normalize according to the most important need, could be not stable, as the most important need may change in different population of users.

In case of inconsistency, if possible, it is preferable to discuss this with responders. In a user need elicitation study, especially when patients are involved, it is more appropriate if the elicitor is there during the elicitation (by person or in videoconference) because patients may need in any moment clarifications about the hierarchy or the method. Being there, it will not be difficult to discuss with responders the consistency of their answers, which often are due to distraction or lousing of interest. Alternative methods would have the same effect of low pass smoothing filters, reducing differences across responders' prioritization of needs. Finally, it is recommended to pay particular attention to the first questionnaire sections, where the responders are particularly exposed to the risk of give inconsistent answers due to the reduced experience with the AHP, and in the last sections of the questionnaires, were the users are particularly exposed to the risk of giving inconsistent answers, because tired. Figure 8 shows how the percentage of responders giving consistent answers $(\mathrm{CR}<0.1)$ in each one of the 21 sections of the questionnaire(Pecchia, Bath et al. 2011), at the first round of the survey, before inconsistencies were discussed. It is possible to observe that the percentage of responders giving consistent answers increased in the sections 9-12, to drop down at the end (section 21), with the exception of the section 20.

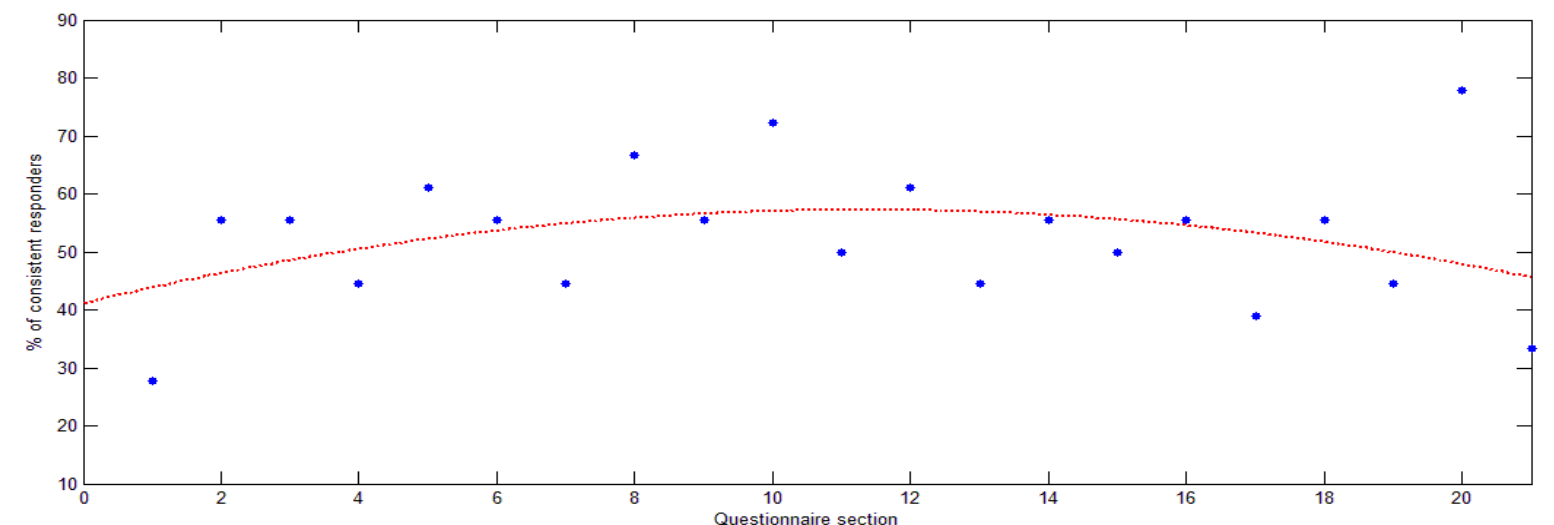

Figure 8. Percentage of users giving consistent answers (CR<0.1), before discussing consistency.

Regarding the data pooling, it have to be remarked that in user elicitation study differences in opinions are what we are looking for. For this reason, to perform the arithmetic mean on the 
eigenvectors components can be more useful than performing the geometric means on the judgment matrices. This is because the former method allows calculating also the standard deviation among priorities given by each user of by significant groups of them. This is crucial to prove the statistical significance of different opinions among groups of users.

In other words, the computation of the geometric mean of the judgments matrix works well, but masks differences across responders' preferences, which represent a very valuable bit of information. In previous studies on how HCPs prioritize risk factors for fall in the elderly (Pecchia, Bath et al. 2010; Pecchia, Bath et al. 2011), it resulted clearly that geriatric doctors prioritization was significantly different form the one done from physiotherapist.. From our point of view, this was because they see the clinical problem in different moments of the care (emergency versus rehabilitation). This is not a problem, but evidencing this different view was one of the main results of the study. In the same study, the minimum consensus was around one risk factor (incontinence), which was considered the most important from some HCP and the last form others, independently from their specialization. This inspired the conclusion that more studies designed to prove the importance of this factor were timely and due. The identification of grey areas were there is limited consensus is paramount important in medicine, were $\mathrm{HCP}$ are committed to follow international guidelines according to the best available evidence.

\section{Conclusions}

Everyday HCP are required to use their personal experience and knowledge to interpret EBM results and apply these in specific cases, according to patients clinical conditions, history and specific needs. This can be not considered at all, or can be faced modelling the decision maker and the user needs as a crucial part of the decisional process. AHP allows to quantify qualitative information and to include this in healthcare decision-making processes.

AHP is particularly effective in eliciting user needs. Its effectiveness can be improved adapting the methods to the responders in consideration of their limited experience with mathematical methods and considering the heterogeneous background of responders, some of which may be emotionally involved in the subject of the elicitation.

This paper, after introducing three case studies in which AHP was effectively applied to user need elicitation, generalized those methodological adaptation that the author consider paramount important to elicit user needs, especially when in the study are involved experts not skilled in the use of the AHP method.

\section{REFERENCES}

Baral, V. R. and J. O. Hourihane (2005). "Food allergy in children." Postgrad Med J 81(961): 693701.

Bock, S. A., A. Munoz-Furlong, et al. (2001). "Fatalities due to anaphylactic reactions to foods." Journal of Allergy and Clinical Immunology 107(1): 191-193.

Bracale, U., M. Rovani, et al. (2011). "Totally laparoscopic gastrectomy for gastric cancer: Metaanalysis of short-term outcomes." Minim Invasive Ther Allied Technol.

Bracale, U., M. Rovani, et al. (2012). "Which is the best laparoscopic approach for inguinal hernia repair: TEP or TAPP? A network meta-analysis " Surgical endoscopy Epub ahead of print. 
Bracale, U., M. Rovani, et al. (2012). "Beneficial effects of fibrin glue (Quixil) versus Lichtenstein conventional technique in inguinal hernia repair: a randomized clinical trial." Hernia.

Chatburn, R. L. and F. P. Primiano, Jr. (2001). "Decision analysis for large capital purchases: how to buy a ventilator." Respir Care 46(10): 1038-1053.

Choo, K. and A. Sheikh (2007). "Action plans for the long-term management of anaphylaxis: systematic review of effectiveness." Clinical and Experimental Allergy 37(7): 1090-1094.

Craven, M., L. Pecchia, et al. (2013). User requirements for image-guided navigation in spinal surgery. World Congress on Medical Physics and Biomedical Engineering May 26-31, 2012, Beijing, China, Springer.

Cummings, A. J., R. C. Knibb, et al. (2010). "Management of nut allergy influences quality of life and anxiety in children and their mothers." Pediatric Allergy and Immunology 21(4): 586-594.

Danner, M., J. M. Hummel, et al. (2011). "Integrating patients' views into health technology assessment: Analytic hierarchy process (AHP) as a method to elicit patient preferences." International Journal of Technology Assessment in Health Care 27(4): 369-375.

Dolan, J. G. (2010). "Multi-criteria clinical decision support: A primer on the use of multiple criteria decision making methods to promote evidence-based, patient-centered healthcare." Patient 3(4): 229248.

Ewan, P. W. (1998). "ABC of allergies - Anaphylaxis." British Medical Journal 316(7142): 14421445.

Gupta, R., A. Sheikh, et al. (2007). "Time trends in allergic disorders in the UK." Thorax 62(1): 9196.

Hummel, M. J. M., W. van Rossum, et al. (2000). "Assessing medical technologies in development A new paradigm of medical technology assessment." International Journal of Technology Assessment in Health Care 16(4): 1214-1219.

Leys, M. (2003). "Health care policy: qualitative evidence and health technology assessment." Health Policy 65(3): 217-226.

Liberatore, M. J. and R. L. Nydick (2008). "The analytic hierarchy process in medical and health care decision making: A literature review." European Journal of Operational Research 189(1): 194-207.

Money, A. G., J. Barnett, et al. (2012). "Patient perceptions of epinephrine auto-injectors: exploring barriers to use." Scand J Caring Sci.

Muraro, A., G. Roberts, et al. (2007). "The management of anaphylaxis in childhood: position paper of the European academy of allergology and clinical immunology." Allergy 62(8): 857-871.

Pecchia, L., P. A. Bath, et al. (2010). "Web-based system for assessing risk factors for falls in community-dwelling elderly people using the analytic hierarchy process." International Journal of the Analytic Hierarchy Process 2(2): 135-157.

Pecchia, L., P. A. Bath, et al. (2011). "Analytic Hierarchy Process (AHP) for Examining Healthcare Professionals' Assessments of Risk Factors The Relative Importance of Risk Factors for Falls in Community-dwelling Older People." Methods Inf Med 50(5): 435-444. 
Pecchia, L., P. A. Bath, et al. (2011). "Analytic Hierarchy Process (AHP) for examining healthcare professionals' assessments of risk factors. The relative importance of risk factors for falls in community-dwelling older people." Methods Inf Med 50(5): 435-444.

Pecchia, L., P. A. Bath, et al. (2011). The use of analytic hierarchy process for the prioritization of factors affecting wellbeing in elderly. International Symposium on Analytic Hierarchy Process (ISAHP). Sorrento, Naples, Italy: 4.

Pecchia, L. and M. Craven (2013). Early stage Health Technology Assessment (HTA) of biomedical devices. The MATCH experience. World Congress on Medical Physics and Biomedical Engineering May 26-31, 2012, Beijing, China, Springer.

Pecchia, L., J. L. Martin, et al. (2013). Application of Analytic Hierarchy Process for User Needs Elicitation: A Preliminary Study on a Device for Auto-injection of Epinephrine. Health Information Science, Springer: 258-264.

Pecchia, L., J. L. Martin, et al. (2013). "User needs elicitation via analytic hierarchy process (AHP). A case study on a Computed Tomography (CT) scanner." BMC Med Inform Decis Mak 13(1): 2.

Pecchia, L., J. L. Martin, et al. (2013). "User needs elicitation via analytic hierarchy process (AHP). A case study on a Computed Tomography (CT) scanner." BMC Medical Informatics and Decision Making 13(1): 2.

Pumphrey, R. (2008). "When should self-injectible epinephrine be prescribed for food allergy and when should it be used?" Curr Opin Allergy Clin Immunol 8(3): 254-260.

Saaty, T. L. (1988). What is the analytic hierarchy process?, Springer.

Sheikh, A., J. Hippisley-Cox, et al. (2008). "Trends in national incidence, lifetime prevalence and adrenaline prescribing for anaphylaxis in England." J R Soc Med 101(3): 139-143.

Simons, F. E. R. (2004). "First-aid treatment of anaphylaxis to food: Focus on epinephrine." Journal of Allergy and Clinical Immunology 113(5): 837-844.

Sloane, E. B., M. J. Liberatore, et al. (2003). "Using the analytic hierarchy process as a clinical engineering tool to facilitate an iterative, multidisciplinary, microeconomic health technology assessment." Computers \& Operations Research 30(10): 1447-1465.

Turri, J. J. (1988). "Program eases decision making." Health Prog 69(8): 40-44. 